\title{
Nuclear Electromagnetic Generator: Introduction in Neutron Algebra and Elements of Neutron Kinetics
}

\author{
V. Yu. Tertychny-Dauri \\ Department of Physics and Engineering, Saint-Petersburg National Research University of Information Technologies, Mechanics \\ and Optics, Saint-Petersburg, Russia \\ Email: tertychny-dauri@mail.ru
}

How to cite this paper: Tertychny-Dauri, V.Yu. (2018) Nuclear Electromagnetic Generator: Introduction in Neutron Algebra and Elements of Neutron Kinetics. Open Access Library Journal, 5: e4670

https://doi.org/10.4236/oalib.1104670

Received: May 21, 2018

Accepted: June 23, 2018

Published: June 26, 2018

Copyright $\odot 2018$ by author and Open Access Library Inc.

This work is licensed under the Creative Commons Attribution International License (CC BY 4.0).

http://creativecommons.org/licenses/by/4.0/

\begin{abstract}
In the work, the problem of neutron kinetics in general view taking into account the all groups of delayed neutrons is solved. Conception of the matrix of complete neutron kinetics is introduced and are determined its properties. In the task about a finding of overt form for the solution of kinetic equations, the separation method and the reducing method of integrodifferential task to integral task with the aid of matched theorem are used. Diffusion of neutrons in the toroidal nuclear electric generator (nuclegen) is investigated also. Important conclusion about the practically complete absence of the diffusion of neutrons and charged fission particles (ionites) through the external boundary under toroidal motion is substantiated when solving of corresponding diffusion equation with the aid of Fourier's standard separation method.
\end{abstract}

\section{Subject Areas}

Modern Physics, Nuclear Physics, Theoretical Physics

\section{Keywords}

Neutron Kinetics, Kinetics of Neutron Multiplication by the Nuclear Fission, Diffusion of Neutrons

\section{Introduction}

This paper is devoted to the solving of a number of important problems of neutron kinetics. The most significance here is that problems connecting with questions of neutron multiplication in the nuclear fission process are presented. Special interest induces the kinetic analysis of delayed neutrons in the general neutrons balance. In the work, determining influence of delayed neutrons on the 
average life time of one neutron generation in considered nuclear device (nuclegen) is noted. Author's works [1], [2], [3], [4] also are devoted to the theoretical grounds of the nuclegen creation.

With the implementation of the project on the creation of toroidal nuclear electric generator the important is the question about the diffusion of neutrons and the question about spreading of diffusing the flow (flex) of neutrons in this nuclear device [5], [6], [7].

The main attention in Sections 2-5 is given to the solving of neutron kinetics problems in the general view. With that purpose the conception of the matrix of complete neutron kinetics is introduced. Given matrix allows finding an overt form for solutions of kinetic equations by means of employment: 1) of the separation method for kinetic equations and 2) of the reducing method of integrodifferential equations equivalent for kinetic equations to corresponding integral equations and its solutions.

In Sections 6-8 the problem of neutron diffusion in a nuclegen is investigated. It is succeeded in solving of diffusion equation with the aid of Fourier's separation method of variables. Obtained solutions allow confirming about the practically complete absence of some penetrating diffusion.

\section{Problem of Neutron Kinetics. Basic Concepts and Preliminary Clarifications}

In the process of nuclear fission two chief neutron groups are formed. In the first one of these groups neutrons are emitted almost instantaneously (within $10^{-14} \mathrm{~s}$ from the very process of fission) and because of that are called prompt neutrons. Prompt neutrons make about $99.36 \%$ of the total quantity of emitted neutrons. The second group is formed of the delayed neutrons, which come from the splinters of fission after their radioactive $\beta$-decay after some time, measured by fractions and tens of seconds.

10 known precursors of delayed neutrons can be pointed out [5], [6] and besides each one of them produces neutrons with a corresponding period of half-life and energy, which is lower than that of prompt neutrons. Of these precursors six [5], [6], [7], having the largest output of delayed neutrons, are usually selected to be taken into account due to difficulty of analysis.

Under a stationary operating condition of a nuclear device (reactor, generator-nuclegen) a critical state of a system, when the multiplication factor of neutrons $k=1$, corresponds with a self-sustaining fission reaction. Understandably during operation of the device within the limits of this condition the factor $k$ is to be changing within the neighbourhood of one. Because of this, a new coefficient is introduced to describe the operation state of a nuclear device

$$
\pm \Delta k=k-1
$$

and it is called excess multiplication factor or excess reactivity. It is obvious that the device remains in the critical state if $\Delta k=0$, in supercritical if $\Delta k>0$ and in subcritical if $\Delta k<0$. 
Apart from the values $k$ and $\Delta k$ in order to characterize state of the active zone a value

$$
\delta k=\frac{ \pm \Delta k}{k}=\frac{k-1}{k}
$$

is often used, which is called reactivity and signifies the fraction of the change of neutron density in a new generation.

The average neutron generation time $l$ is the time from the moment of the neutrons formation during fission to the moment of their absorption (with the challenge of a new act of fission or ultimately output from the reaction). The rate of increase or decrease of neutron density $n$ (number of neutrons $N$ in a unity of volume) in the active zone depends on the average generation time $l$ of neutrons. Naturally, the less is the generation time $l$ until capture with fission, the more is the rate of increase of density $n$, that is

$$
\frac{\mathrm{d} n}{\mathrm{~d} t} \sim \frac{1}{l}
$$

This expression can be given a more precise form. Indeed, in the next generation at the presence of excess reactivity the neutron density is to be equal $n(1+\Delta k)$. Because of this, the rate of change of neutron density without taking delayed neutrons into account obeys the equation

$$
\frac{\mathrm{d} n}{\mathrm{~d} t}=\frac{n(1+\Delta k)-n}{l},
$$

from which $n=n_{0} \exp (\Delta k \cdot t / l)$, in which $n_{0}$ is neutron density at the initial time.

In kinetic analysis of nuclear devices the concept of period $T$ of a given device is introduced, so that $t=T$ is the time, in the duration of which the neutron density $n$ changes $e$ times, that is

$$
\frac{n}{n_{0}}=e=\mathrm{e}^{\Delta k \cdot T / l}
$$

and, consequently, $T=l / \Delta k$.

If delayed neutrons are to be taken into account in the general kinetics equation, then the following neutron balance is achieved. Specifically, for $m=6$ groups of delayed neutrons the rate of change of neutron density $n=n(t)$ equals

$$
\frac{\mathrm{d} n}{\mathrm{~d} t}=\frac{k n}{l}-\frac{n}{l}-\frac{k n \beta}{l}+\sum_{i=1}^{m} \lambda_{i} C_{i}+S,
$$

in which the first summand in the right-hand side of the Equation (1) signifies the rate of formation of all neutrons by fission, the second one signifies the rate of neutron loss, the third one signifies the rate of formation of precursors, the fourth one signifies the rate of formation of delayed neutrons out of these precursors, the fifth one signifies the intensity $S$ of an external source of neutrons.

Here $\beta$ is the portion of delayed neutrons, $1-\beta$ is the portion of prompt neutrons, $\lambda_{i}=1 / l_{i}$ is a constant of decay of fission splinters (precursor nuclei), which emit delayed neutrons of group number $i, l_{i}$ is the lifetime of these pre- 
cursor nuclei, $C_{i}$ is the number (concentration) of precursor nuclei of delayed neutrons of group number $i$.

The quantity $C_{i}=C_{i}(t)$ satisfies the equation

$$
\frac{\mathrm{d} C_{i}}{\mathrm{~d} t}=-\lambda_{i} C_{i}+\frac{k n \beta_{i}}{l}, \quad i=\overline{1, m},
$$

in which $\beta_{i}$ is the portion of delayed neutrons of each group. In the Equation (2) the first summand in the right-hand side gives the decrease of the number of precursor nuclei due to decay, and the second one gives their formation. Also notable is that for the substantiation of the system of Equations (1) and (2) it is supposed that the lifetime of daughter delayed neutrons and their precursor nuclei coincides.

Obviously, Equation (1) can be written dawn in the form

$$
\frac{\mathrm{d} n}{\mathrm{~d} t}=\frac{[k(1-\beta)-1] n}{l}+\sum_{i=1}^{m} \lambda_{i} C_{i},
$$

if it is considered that $S \equiv 0$. The system of Equations (2) and (3) describes the behavior of neutron density in the time and because of that it may be admitted as the most important in the task of kinetic neutron research. From that system it is clearly seen that the number (concentration) of precursor nuclei $C_{i}(t), i=\overline{1, m}$, forms feedback in regards to neutron density.

For simplification and greater visualization of the calculations an averaged approximation of one group of delayed neutrons with an averaged lifetime $l_{*}$ is typically used for kinetic analysis, in which

$$
l_{*}=\frac{\sum_{i=1}^{m} l_{i} \beta_{i}}{\beta}, \quad \beta=\sum_{i=1}^{m} \beta_{i} .
$$

Then, obviously, the average lifetime of a generation of neutrons $\bar{l}$ can be found as a sum of average lifetimes of delayed and prompt neutrons taking into account their portions in the total composition of neutrons:

$$
\bar{l}=\beta l_{*}+(1-\beta) l=\sum_{i=1}^{m} l_{i} \beta_{i}+(1-\beta) l .
$$

In the expression (4) there is an estimation

$$
(1-\beta) l \ll \sum_{i=1}^{m} l_{i} \beta_{i}
$$

from which follows an important ascertaining: an average lifetime of one neutron generation in a nuclear device is practically defined by the lifetime of delayed neutrons.

\section{Matrix of Full Neutron Kinetics}

Write a system of differential Equations (2) and (3) in vector-matrix form

$$
\dot{x}(t)=A x(t), \quad x(t) \in \mathbf{R}^{m+1}, \quad x(0)=x_{0},
$$

where designations are introduced 


$$
\begin{gathered}
x=\left(\begin{array}{c}
n \\
C_{1} \\
C_{2} \\
\vdots \\
C_{m}
\end{array}\right), \quad A=\left(\begin{array}{ccccc}
\varphi_{0} & \lambda_{1} & \lambda_{2} & \cdots & \lambda_{m} \\
\varphi_{1} & -\lambda_{1} & 0 & \cdots & 0 \\
\varphi_{2} & 0 & -\lambda_{2} & \cdots & 0 \\
\vdots & \vdots & \vdots & \ddots & \vdots \\
\varphi_{m} & 0 & 0 & \cdots & -\lambda_{m}
\end{array}\right), \\
\varphi_{0}=\frac{k(1-\beta)-1}{l}, \quad \varphi_{i}=\frac{k \beta_{i}}{l}, \quad i=\overline{1, m} .
\end{gathered}
$$

For a constant square matrix $A$ of dimension $m+1$ the Equation (5) has a single solution $x(t)=\mathrm{e}^{A t} \cdot x_{0}$. However that solution cannot be used to write down the explicit form of all the solution components $x$ because of the presence of a matrix exponent.

Matrix $A$ can be called a matrix of full neutron kinetics. The calculation algorithm of the determinant of this matrix is adduced.

Theorem 1. Determinant of matrix $A$ is calculated by the formula

$$
\operatorname{det} A=(-1)^{m} \prod_{i=1}^{m} \lambda_{i} \cdot \sum_{j=0}^{m} \varphi_{j}
$$

Proof. With the goal of deriving the correlation (6) we shall utilize the expansion method of the determinant on columns with highest number of zeroes and reduction to calculation of lesser order determinants of triangular form. Then, starting with the second column, on the first step we get

$$
\operatorname{det} A=-\lambda_{1} \operatorname{det} A_{1}^{(0)}-\lambda_{1} \operatorname{det} A_{1}^{(1)} \text {, }
$$

in which

$$
A_{1}^{(0)}=\left(\begin{array}{ccccc}
\varphi_{1} & 0 & 0 & \cdots & 0 \\
\varphi_{2} & -\lambda_{2} & 0 & \cdots & 0 \\
\varphi_{3} & 0 & -\lambda_{3} & \cdots & 0 \\
\vdots & \vdots & \vdots & \ddots & \vdots \\
\varphi_{m} & 0 & 0 & \cdots & -\lambda_{m}
\end{array}\right), A_{1}^{(1)}=\left(\begin{array}{ccccc}
\varphi_{0} & \lambda_{2} & \lambda_{3} & \cdots & \lambda_{m} \\
\varphi_{2} & -\lambda_{2} & 0 & \cdots & 0 \\
\varphi_{3} & 0 & -\lambda_{3} & \cdots & 0 \\
\vdots & \vdots & \vdots & \ddots & \vdots \\
\varphi_{m} & 0 & 0 & \cdots & -\lambda_{m}
\end{array}\right),
$$

that is

$$
\operatorname{det} A=(-1)^{m} \varphi_{1} \lambda_{1} \lambda_{2} \cdots \lambda_{m}-\lambda_{1} \operatorname{det} A_{1}^{(1)} .
$$

Following this, in the equality (7) on 2-nd step the $\operatorname{det} A_{1}^{(1)}$ is again expanded along the elements of the second column:

$$
\operatorname{det} A_{1}^{(1)}=-\lambda_{2} \operatorname{det} A_{2}^{(0)}-\lambda_{2} \operatorname{det} A_{2}^{(1)},
$$

in which

$$
A_{2}^{(0)}=\left(\begin{array}{ccccc}
\varphi_{2} & 0 & 0 & \cdots & 0 \\
\varphi_{3} & -\lambda_{3} & 0 & \cdots & 0 \\
\varphi_{4} & 0 & -\lambda_{4} & \cdots & 0 \\
\vdots & \vdots & \vdots & \ddots & \vdots \\
\varphi_{m} & 0 & 0 & \cdots & -\lambda_{m}
\end{array}\right), A_{2}^{(1)}=\left(\begin{array}{ccccc}
\varphi_{0} & \lambda_{3} & \lambda_{4} & \cdots & \lambda_{m} \\
\varphi_{3} & -\lambda_{3} & 0 & \cdots & 0 \\
\varphi_{4} & 0 & -\lambda_{4} & \cdots & 0 \\
\vdots & \vdots & \vdots & \ddots & \vdots \\
\varphi_{m} & 0 & 0 & \cdots & -\lambda_{m}
\end{array}\right),
$$

This means that 


$$
\operatorname{det} A_{1}^{(1)}=\varphi_{2}\left(-\lambda_{2}\right)\left(-\lambda_{3}\right) \cdots\left(-\lambda_{m}\right)-\lambda_{2} \operatorname{det} A_{2}^{(1)} .
$$

Substituting the expression (8) into the formula (7) we get

$$
\operatorname{det} A=(-1)^{m} \lambda_{1} \lambda_{2} \cdots \lambda_{m}\left(\varphi_{1}+\varphi_{2}\right)+\left(-\lambda_{1}\right)\left(-\lambda_{2}\right) \operatorname{det} A_{2}^{(1)} .
$$

Continuing this process and successively reducing it to calculation of $\operatorname{det} A_{2}^{(1)}, \operatorname{det} A_{3}^{(1)}$ and etc we come to the equality (6) on the final $m$-th step:

$$
\begin{aligned}
\operatorname{det} A= & (-1)^{m} \lambda_{1} \lambda_{2} \cdots \lambda_{m}\left(\varphi_{1}+\varphi_{2}+\cdots+\varphi_{m-1}\right)+\left(-\lambda_{1}\right) \cdots\left(-\lambda_{m-1}\right)\left(-\lambda_{m}\right) \varphi_{m} \\
& +\left(-\lambda_{1}\right) \cdots\left(-\lambda_{m-1}\right)\left(-\lambda_{m}\right) \varphi_{0} .
\end{aligned}
$$

The Theorem 1 is proven.

Consequence. The expressions for $\varphi_{j}, j=\overline{0, m}$ are substituted into formula (6). Then, obviously, we get

$$
\sum_{j=0}^{m} \varphi_{j}=\frac{k-1}{l}, \text { in which } \varphi_{i}=\frac{k \beta_{i}}{l}, \quad \sum_{i=1}^{m} \beta_{i}=\beta .
$$

From here an important conclusion ensues that the matrix $A$ is degenerate for $k=1$, that is, for the critical state of the nuclear device. In that case there is a stationary operating regime $x=x_{0}, \dot{x}=A x_{0}=0 \quad$ (rate of change of neutron density and their precursors equals zero). Then Equations (2) and (3) take on the form $(k=1)$ respectively:

$$
\begin{gathered}
0=-\lambda_{i} C_{i 0}+\frac{\beta_{i} n_{0}}{l}, \\
0=-\frac{\beta n_{0}}{l}+\sum_{i=1}^{m} \lambda_{i} C_{i 0},
\end{gathered}
$$

in which $n_{0}=n(0), C_{i 0}=C_{i}(0), i=\overline{1, m}$. From the first equation $C_{i 0}=\beta_{i} n_{0} /\left(\lambda_{i} l\right)$. If this value of $C_{i 0}$ is substituted into the second equation of the system (9), then we come to an identity, which proves the rightness of the substantiation of initial conditions data of the process of nuclear (neutron) kinetics.

Considered further is a task of finding the explicit form of the solution of the Equation (5) while accounting for the results of Theorem 1. Apparently all elements of the matrix exponent $\mathrm{e}^{A t}$ need to be found for that. As it is known (refer, for instance, to the works [8], [9]), this task is solved in a general case by means of finding eigenvalues and eigenvectors of the matrix $A$.

Various eigenvalues of matrix $A$ are designated by $\alpha_{j}, j=\overline{0, m}$. The left-hand side of the characteristic equation $\operatorname{det}(A-\alpha I)=0$, where $I$ is the identity matrix, for determination of $\alpha_{j}$ takes the form of a polynomial $P_{m+1}(\alpha)$ by $\alpha$ of degree $m+1$, the coefficients of which (and thus also the roots) are rather difficult to calculate. As a vivid example polynomials for various groups of delayed neutrons $P_{2}(\alpha), P_{3}(\alpha), P_{4}(\alpha)$ are written down.

1) One-group kinetic model:

$$
P_{2}(\alpha)=(-1)^{1}\left[\lambda_{1}\left(\varphi_{0}+\varphi_{1}\right)+\left(\varphi_{0}-\lambda_{1}\right) \alpha-\alpha^{2}\right] .
$$

2) Two-groups kinetic model: 


$$
\begin{aligned}
P_{3}(\alpha)= & (-1)^{2}\left\{\lambda_{1} \lambda_{2}\left(\varphi_{0}+\varphi_{1}+\varphi_{2}\right)+\left[\left(\lambda_{1}+\lambda_{2}\right) \varphi_{0}+\lambda_{1} \varphi_{1}+\lambda_{2} \varphi_{2}-\lambda_{1} \lambda_{2}\right] \alpha\right. \\
& \left.+\left[\varphi_{0}-\left(\lambda_{1}+\lambda_{2}\right)\right] \alpha^{2}-\alpha^{3}\right\} .
\end{aligned}
$$

3) Three-groups kinetic model:

$$
\begin{aligned}
P_{4}(\alpha)= & (-1)^{3}\left\{\lambda_{1} \lambda_{2} \lambda_{3}\left(\varphi_{0}+\varphi_{1}+\varphi_{2}+\varphi_{3}\right)+\left[\left(\lambda_{1} \lambda_{2}+\lambda_{1} \lambda_{3}+\lambda_{2} \lambda_{3}\right) \varphi_{0}\right.\right. \\
& \left.+\lambda_{1}\left(\lambda_{2}+\lambda_{3}\right) \varphi_{1}+\lambda_{2}\left(\lambda_{1}+\lambda_{3}\right) \varphi_{2}+\lambda_{3}\left(\lambda_{1}+\lambda_{2}\right) \varphi_{3}-\lambda_{1} \lambda_{2} \lambda_{3}\right] \alpha \\
& +\left[\left(\lambda_{1}+\lambda_{2}+\lambda_{3}\right) \varphi_{0}+\lambda_{1} \varphi_{1}+\lambda_{2} \varphi_{2}+\lambda_{3} \varphi_{3}-\left(\lambda_{1} \lambda_{2}+\lambda_{1} \lambda_{3}+\lambda_{2} \lambda_{3}\right)\right] \alpha^{2} \\
& \left.+\left[\varphi_{0}-\left(\lambda_{1}+\lambda_{2}+\lambda_{3}\right)\right] \alpha^{3}-\alpha^{4}\right\} .
\end{aligned}
$$

From here the search for an analytical solution with the help of the algorithm of finding roots of the equation $P_{m+1}(\alpha)=0$ in a general case is revealed as prospectless. In the meantime, the situation is not as hope less and changes in a radical way if the system Equation (2) and scalar Equation (3) are separated.

\section{Separation Method. Integrodifferential Task}

System (2) can be rewritten in the form

$$
\begin{gathered}
\dot{y}(t)=B y(t)+n(t) \varphi, \quad y(t), \varphi \in \mathbf{R}^{m}, \quad y(0)=y_{0}, \\
y=\left(\begin{array}{c}
C_{1} \\
C_{2} \\
\vdots \\
C_{m}
\end{array}\right), \varphi=\left(\begin{array}{c}
\varphi_{1} \\
\varphi_{2} \\
\vdots \\
\varphi_{m}
\end{array}\right), B=\left(\begin{array}{cccc}
-\lambda_{1} & 0 & \cdots & 0 \\
0 & -\lambda_{2} & \cdots & 0 \\
\vdots & \vdots & \ddots & \vdots \\
0 & 0 & \cdots & -\lambda_{m}
\end{array}\right) .
\end{gathered}
$$

As a reminder, the initial conditions $y_{i}(0)=C_{i 0}, i=\overline{1, m}$ are defined by the expressions (9).

Integrating the Equation (10), we get

$$
y(t)=\mathrm{e}^{B t} \cdot y_{0}+\int_{0}^{t} \mathrm{e}^{B(t-s)} n(s) \varphi \mathrm{d} s .
$$

Because in the solution (11) the value $n(t)=x_{1}(t)$ is scalar, and the vector $\varphi$ is constant, we have

$$
y(t)=\mathrm{e}^{B t}\left[y_{0}+\left(\int_{0}^{t} n(s) \mathrm{e}^{-B s} \mathrm{~d} s\right) \varphi\right] .
$$

In the expression (12) the matrix exponent $\mathrm{e}^{B t}$ is very simply found in the form

$$
\mathrm{e}^{B t}=\left(\begin{array}{cccc}
\mathrm{e}^{-\lambda_{1} t} & 0 & \cdots & 0 \\
0 & \mathrm{e}^{-\lambda_{2} t} & \cdots & 0 \\
\vdots & \vdots & \ddots & \vdots \\
0 & 0 & \cdots & \mathrm{e}^{-\lambda_{m} t}
\end{array}\right)
$$

and that means, that the solution (12) can be written down element wise:

$$
y_{i}(t)=C_{i}(t)=y_{i 0} \mathrm{e}^{-\lambda_{i} t}+\varphi_{i} \int_{0}^{t} n(s) \mathrm{e}^{\lambda_{i}(s-t)} \mathrm{d} s .
$$

Then the obtained values $C_{i}(t)(13)$ are substituted in the Equation (3), and we get 


$$
\dot{n}(t)=\varphi_{0} n(t)+\sum_{i=1}^{m} \lambda_{i} C_{i 0} \mathrm{e}^{-\lambda_{i} t}+\sum_{i=1}^{m} \lambda_{i} \varphi_{i} \int_{0}^{t} n(s) \mathrm{e}^{\lambda_{i}(s-t)} \mathrm{d} s .
$$

Obviously, this equation is representable in the form of a linear nonhomogeneous integrodifferential Volterra equation of 1-st order

$$
\dot{n}(t)=\varphi_{0} n(t)+\int_{0}^{t} n(s) F_{1}(s, t) \mathrm{d} s+F_{2}(t),
$$

where for the known functions of time $F_{1}(s, t)$ and $F_{2}(t)$ designations are introduced $\left(f_{i}(t)=\mathrm{e}^{\lambda_{i}(t)}\right)$ :

$$
\begin{gathered}
F_{1}(s, t)=\sum_{i=1}^{m} \lambda_{i} \varphi_{i} \mathrm{e}^{\lambda_{i}(s-t)}=\sum_{i=1}^{m} \lambda_{i} \varphi_{i} f_{i}(s) f_{i}(-t), \\
F_{2}(t)=\sum_{i=1}^{m} \lambda_{i} C_{i 0} \mathrm{e}^{-\lambda_{i} t}=\sum_{i=1}^{m} \lambda_{i} C_{i 0} f_{i}(-t) .
\end{gathered}
$$

Integrodifferential equations of the form (14) apparently have first appeared in the tasks of mathematical biology (20-s and 30-s of 20-th century: Verhulst's task about populations and competition within a species, Lotka-Volterra task about "predator-prey" type associations and etc. [10]). A feature of these equations is that all of them are caused by various effects of delay. Thus it is not surprising that the presence of delayed neutrons in the process of nuclear fission also caused a corresponding integrodifferential equation in its wake, in which the effect of the delay is given with the help of the function $F_{1}(s, t)$.

For the solution of the Equation (14) one of the approximate methods can be utilized, for instance, one of quadratures, one of successive approximations and etc [11]. The method of reduction of the initial equation to an integral one with consequent solution through a resolvent kernel is among the more redical analytical methods of solution of integrodifferential equations.

\section{Method of Reduction to an Integral Task}

However in our case the reduction of the initial integrodifferential equation to an integral equation leads to big difficulties due to the presence of three constituents of various differential dimensions at once: $\dot{n}(t), n(t)$ and $\int_{0}^{t} n(s) \mathrm{d} s$. Nonetheless, let's try to utilize one of the variants of the method of reduction [11] with the help of a fitting change of variables.

Let's designate

$$
n(t)=\int_{0}^{t} H(t) \sigma(s) \mathrm{d} s, \quad \dot{n}(t)=\int_{0}^{t} \dot{H}(t) \sigma(s) \mathrm{d} s+H(t) \sigma(t),
$$

in which $\sigma(t)$ is the new sought function, $H(t)$ is a function of a homogenous kernel, which is hereinafter selected from certain conditions of solvability of the equations. Substituting all of these expressions into the Equation (14) we get

$$
\begin{aligned}
& \int_{0}^{t} \dot{H}(t) \sigma(s) \mathrm{d} s+H(t) \sigma(t) \\
& =\varphi_{0} \int_{0}^{t} H(t) \sigma(s) \mathrm{d} s+\int_{0}^{t} F_{1}(s, t)\left(\int_{0}^{s} H(s) \sigma(v) \mathrm{d} v\right) \mathrm{d} s+F_{2}(t),
\end{aligned}
$$


from which

$$
\int_{0}^{t}\left[\dot{H}(t)-\varphi_{0} H(t)\right] \sigma(s) \mathrm{d} s+H(t) \sigma(t)-\int_{0}^{t} F_{1}(s, t)\left(\int_{0}^{s} H(s) \sigma(v) \mathrm{d} v\right) \mathrm{d} s=F_{2}(t) .
$$

Let's further designate

$$
M(t)=\dot{H}(t)-\varphi_{0} H(t), \quad \theta(t)=\int_{0}^{t} M(t) \sigma(s) \mathrm{d} s+H(t) \sigma(t)
$$

and write the Equation (15) down in the form of a linear Volterra integral equation of the second kind:

$$
\theta(t)-\int_{0}^{t} N(s, t) \theta(s) \mathrm{d} s=F_{2}(t),
$$

in which hereinafter the kernel $N(s, t)$ is to be selected appropriately.

Provided identity of the Equations (15) and (16) there is a need for the equality

$$
\begin{aligned}
& \int_{0}^{t} N(s, t)\left(\int_{0}^{s} M(s) \sigma(v) \mathrm{d} v+H(s) \sigma(s)\right) \mathrm{d} s \\
& =\int_{0}^{t} F_{1}(s, t)\left(\int_{0}^{s} H(s) \sigma(v) \mathrm{d} v\right) \mathrm{d} s
\end{aligned}
$$

to hold. This correlation holds, in turn, if the following equality of integrands holds:

$$
\begin{aligned}
& N(s, t) \int_{0}^{s} M(s) \sigma(v) \mathrm{d} v+N(s, t) H(s) \sigma(s) \\
& =F_{1}(s, t) \int_{0}^{s} H(s) \sigma(v) \mathrm{d} v,
\end{aligned}
$$

that is, if there might be the equality

$$
N(s, t) H(s) \sigma(s)=\int_{0}^{s}\left(F_{1}(s, t) H(s)-N(s, t) M(s)\right) \sigma(v) \mathrm{d} v,
$$

or

$$
\sigma(s)=\int_{0}^{s}\left(\frac{F_{1}(s, t)}{N(s, t)}-\frac{M(s)}{H(s)}\right) \sigma(v) \mathrm{d} v .
$$

Obviously, in the integral condition (17) the first fraction $F_{1}(s, t) / N(s, t)$ should not depend on $t$. This happens, for example, if the function selected as the function $N(s, t)$ is such

$$
N(s, t)=g(s) F_{1}(s, t),
$$

in which $g(s)$ is a certain known function of time $s$. Thus, we come to a restriction

$$
h(t) \sigma(t)=\int_{0}^{t} \sigma(s) \mathrm{d} s, \quad h(t)=\frac{g(t) H(t)}{H(t)-g(t) M(t)},
$$

from which we get an equation of a linear holonomic differential constraint of 1-st order:

$$
\dot{\sigma}(t)=\mu(t) \sigma(t), \quad \mu(t)=\frac{1-\dot{h}(t)}{h(t)}
$$


This constraint (18) may also be called a regulated one, because in it the functional coefficient $\mu(t)$ may be selected by some purposeful way.

The simplest and most natural case of the solution of the Equation (18) is considered, in which $\mu(t)=\mu=$ const. We have $\sigma(t)=\sigma_{0} \exp (\mu t)$, in which $\sigma_{0}=\sigma(0)$. In connection with the presence of a series of new introduced functions (which are supposed to be continuously everywhere differentiable with respect to all arguments) and of the corresponding equations an important question of their solvability arises. The following statement in the form of the Theorem of matching provides an answer to it.

Theorem 2. For the selection of a function of time

$$
H(t)=\frac{g(t) \theta(t)}{h(t) \sigma(t)},
$$

in which $g(t)$ is a certain given function $t, t \geq 0, \theta(t)=\theta\left(F_{1}, F_{2}, g, t\right)$ is a known solution of the integral Equation (16), $h(t), \sigma(t)$ are known functions of time, the system of integral dependences (16)-(18) is a concordant one, that is consistent and possessing an unambiguous solution for given initial conditions.

Proof. Let's proceed primarily from the presence of the Equations (16) and (18). On one hand, the function $N(s, t)=g(s) F_{1}(s, t)$ being given fully defines the solution $\theta(t)$ in the integral Equation (16). On the other hand, the solution $\sigma(t)$ in the Equation (18) defines the nature of the dependence

$$
\theta(t)=\int_{0}^{t} M(t) \sigma(s) \mathrm{d} s+H(t) \sigma(t)
$$

if the function of time $H(t)$ is known.

This is handled in the following way. Let $\mu=$ const. Then in the correlation (18) we have

$$
\dot{h}(t)=-\mu h(t)+1, \quad h(t)=\frac{1}{\mu}+\left(h_{0}-1\right) \mathrm{e}^{-\mu t}, \quad h_{0}=h(0) .
$$

Due to the definition of the function $h(t)$ we need to write down

$$
\frac{1}{\mu}+\left(h_{0}-1\right) \mathrm{e}^{-\mu t}=\frac{g(t) H(t)}{H(t)-g(t)\left[\dot{H}(t)-\varphi_{0} H(t)\right]} .
$$

Functions $h(t)$ and $\sigma(t)$ are assumed to be known functions of time. Let's assign the function $g(t)$. Then from the correlation (20) we get

$$
g(t)=\frac{h(t) H(t)}{h(t) M(t)+H(t)}
$$

in which $h(t)$ is an already known function of time. What is more, we have

$$
\theta(t)=[h(t) M(t)+H(t)] \sigma(t),
$$

in which $\theta(t)=\theta\left(F_{1}, F_{2}, g, t\right)$ is a known solution of the integral Equation (16). Substituting the expression for $h(t) M(t)+H(t)$ from the correlation (22) into the dependence (21), we find

$$
g(t)=\frac{h(t) H(t) \sigma(t)}{\theta(t)}
$$


from which the condition of matching (19) follows, quod erat demonstrandum.

In a general summary of the task of solving kinetic equations, it can be concluded that the integral scheme of solution consists of several stages: firstly, we define the function $g(t)$ and the number $\mu$, then we find the functions $N(s, t), \sigma(t)$ and $h(t)$, by them we determine functions $\theta(t)$ and $H(t)$. The found values allow determining the solution of the task, specifically the neutron flux density $n(t)$ and concentration of precursor nuclei $C_{i}(t), i=\overline{1, m}$.

\section{Diffusion Problem of Neutrons and Its Solution by Furier's Method}

It is well known the process of formation of free neutrons inside a fission medium (thereby of formation of charged fission particles) for realization and maintenance of nuclear chain reaction is a decisive significance. Therefore, the question about diffusion of neutrons and charged particles flex over the whole volume of external magnetic reflecting field which created by toroidal nuclear electric generator (nuclegen) is presented sufficiently important.

In critical stationary generator the coefficient of neutron multiplication $k$ from generation to generation doesn't change $(k=1)$. Hence it follows that for any point of active zone we can write the equation of neutron balance

$$
F-L-A-R=0 \text {, }
$$

where $F$ is the "formation", $L$ is the "leakage", $A$ is the "absorption" and $R$ is the "reflection". Here through $R$ in relation (23) the external magnetic pressure is indicated which is making in respect to the neutron flex the reflected and restored function and reflected effect of itself toroidal surface also.

The basic equation for diffusion effects (in this case neutron diffusion) is the Fick's law, which establishes that resulting current (flex) of diffusive substance is proportional to the density gradient of this substance and is directed to the side of region with the lowest density

$$
J=-D \nabla \Phi,
$$

where $\nabla=(\partial / \partial x, \partial / \partial y, \partial / \partial z)$ is the operator of spatial gradient, $J$ is the resultant flex of neutrons, $\Phi=n v$ is the scalar neutron flex, $D$ is the coefficient of diffusion is determined by relation (24). Here $n$ is the number of neutrons in the unit of volume (the spatial density), $v$ is the average neutron density.

We denote the leakage $L$ in Equation (23) through $L$ too. The value $L$ is express itself in the equality

$$
L=-D \Delta \Phi,
$$

where $\Delta=\left(\partial^{2} / \partial x^{2}, \partial^{2} / \partial y^{2}, \partial^{2} / \partial z^{2}\right)$ is the Laplace operator. If to count up that in Equation (23) "formation", "absorption", "reflection" each separately are proportional to the flex $\Phi$ then taking into account relations (24), (25) the Equation (23) can be written for some proportionality coefficient $R$ in the form:

$$
D \Delta \Phi+R \Phi=0,
$$


or

$$
\Delta \Phi+a^{2} \Phi=0,\left.\quad \Phi\right|_{\Gamma} \in \Omega
$$

where $a^{2}=R / D,\left.\Phi\right|_{\Gamma} \in \Omega$ is the boundary condition on the $\Gamma$ from some region $\Omega$ concerning the function $\Phi$. We solve Equation (26) with a given boundary condition (from region $\Omega$ ) into supposition that the flex $\Phi$ is twice continuously differentiable on spatial variables. With that end in view we introduce cylindrical coordinates $(r, \varphi, z)$. We have for the arbitrary function $F(r, \varphi, z)$ :

$$
\Delta F=\frac{1}{r} \frac{\partial}{\partial r}\left(r \frac{\partial F}{\partial r}\right)+\frac{1}{r^{2}} \frac{\partial^{2} F}{\partial \varphi^{2}}+\frac{\partial^{2} F}{\partial z^{2}} .
$$

Owing to the symmetry of toroid the flex doesn't depend on the angular coordinate $\varphi$ and the diffusion problem (26) becomes two-dimensional: $\Phi=\Phi(r, z)$, i.e.

$$
\frac{\partial^{2} \Phi(r, z)}{\partial r^{2}}+\frac{1}{r} \frac{\partial \Phi(r, z)}{\partial r}+\frac{\partial^{2} \Phi(r, z)}{\partial z^{2}}+a^{2} \Phi(r, z)=0
$$

where $r_{1} \leq r \leq r_{2},-r_{0} \leq z \leq r_{0}, r_{0}=\left(r_{2}-r_{1}\right) / 2$. We use the Furier's method variables separation for the solving of Equation (27) supposed that the flex $\Phi(r, z)$ is presented in the form of two functions product as

$$
\Phi(r, z)=R(r) Z(z) .
$$

We substitute expression (28) in the equation of diffusion (27). After termwise division on $R(r) Z(z)$ we receive the equation

$$
\frac{1}{R(r)}\left[\frac{\mathrm{d}^{2} R(r)}{\mathrm{d} r^{2}}+\frac{1}{r} \frac{\mathrm{d} R(r)}{\mathrm{d} r}\right]+a^{2}=-\frac{1}{Z(z)} \cdot \frac{\mathrm{d}^{2} Z(z)}{\mathrm{d} z^{2}} .
$$

The left part of Equation (29) depends only from $r$ and the right-only from $z$. The function (28) can be the solution of Equation (29) as soon as in the case if both parts of this equation are constant by the arbitrary changing of variables $r$ and $z$, i.e. must be carried out the following relations

$$
\begin{gathered}
\frac{1}{R(r)}\left[\frac{\mathrm{d}^{2} R(r)}{\mathrm{d} r^{2}}+\frac{1}{r} \frac{\mathrm{d} R(r)}{\mathrm{d} r}\right]=-a_{r}^{2}, \\
\frac{1}{Z(z)} \cdot \frac{\mathrm{d}^{2} Z(z)}{\mathrm{d} z^{2}}=-a_{z}^{2},
\end{gathered}
$$

where $a_{r}^{2}, a_{z}^{2}$ are positive numbers connected by equality $a^{2}=a_{r}^{2}+a_{z}^{2}$ owing to Equation (29).

\section{Solution of Equation on $R(r)$}

Consider Equation (30). At first we multiply it by $r^{2} R(r)$ :

$$
r^{2} \frac{\mathrm{d}^{2} R(r)}{\mathrm{d} r^{2}}+r \frac{\mathrm{d} R(r)}{\mathrm{d} r}+r^{2} R(r) a_{r}^{2}=0,
$$


and then introduce the new variable $\rho=r a_{r}$. As a result we obtain the Bessel's equation of zero order

$$
\rho^{2} \frac{\mathrm{d}^{2} R(\rho)}{\mathrm{d} \rho^{2}}+\rho \frac{\mathrm{d} R(\rho)}{\mathrm{d} \rho}+\rho^{2} R(\rho)=0 .
$$

The general solution of Equation (32) is presented in the form

$$
R(\rho)=C_{1} J_{0}(\rho)+C_{2} Y_{0}(\rho),
$$

where $C_{1}, C_{2}$ are the arbitrary constants, $J_{0}(\rho), Y_{0}(\rho)$ are the Bessel's functions of zero order of first and second kinds determined with the help of power series

$$
\begin{gathered}
J_{0}(\rho)=1+\sum_{k=1}^{\infty} \frac{(-1)^{k} \rho^{2 k}}{(k !)^{2} 2^{2 k}}, \\
Y_{0}(\rho)=\lim _{n \rightarrow 0} \frac{J_{n}(\rho) \cos n \pi-J_{-n}(\rho)}{\sin n \pi} .
\end{gathered}
$$

Here $J_{-n}(\rho)=(-1)^{n} J_{n}(\rho), J_{n}(\rho)$ is the Bessel's function of $n$-th order with the constant $a^{(n)}$, i.e.

$$
\begin{gathered}
J_{n}(\rho)=a^{(n)} \rho^{n} \sum_{k=0}^{\infty} \frac{(-1)^{k} \rho^{2 k}}{k !(n+1)(n+2) \cdots(n+k) 2^{2 k},} \\
a^{(n)}=\frac{1}{2^{n} \Gamma(n+1)}, \quad \Gamma(n)=\int_{0}^{\infty} \mathrm{e}^{-x} x^{n-1} \mathrm{~d} x,
\end{gathered}
$$

where $\Gamma(n)$ is the Euler gamma-function. It can be shown, that for small $\rho$ :

$$
Y_{0}(\rho) \approx-\frac{2}{\pi} \ln \left(\frac{2}{\gamma \rho}\right),
$$

where $\ln \gamma=C=0.5772 \cdots$ is the Euler's constant. Sometimes the function $Y_{0}(\rho)$ is called the Neumann's (or Weber's) function of zero order. If come back to the variable $r$ then we can write as a result

$$
R(r)=C_{1} J_{0}\left(a_{r} r\right)+C_{2} Y_{0}\left(a_{r} r\right) .
$$

\section{Solution of Equation on $Z(z)$ and the General Solution}

Consider now Equation (31). Its solution is determined easily

$$
Z(z)=C_{3} \sin a_{z} z+C_{4} \cos a_{z} z,
$$

where $C_{3}, C_{4}$ are the arbitrary constants. We take from conditions of symmetry

$$
Z=Z_{0},\left.\quad \frac{\mathrm{d} Z(z)}{\mathrm{d} z}\right|_{z=0}=0 .
$$

Then it is obvious that in solution (34) by conditions (35) we have $C_{3}=0, C_{4}=Z_{0}$ and consequently

$$
Z(z)=Z_{0} \cos a_{z} z
$$


Multiply these two solutions (33) and (36). As a result we obtain the expression for flex (28):

$$
\Phi(r, z)=\left[C_{1} J_{0}\left(a_{r} r\right)+C_{2} Y_{0}\left(a_{r} r\right)\right] Z_{0} \cos a_{z} z
$$

Solution (37) should satisfy the basic boundary condition:

$$
\Phi(r, z)=0, \forall r, z \in T_{\delta},
$$

where

$$
T_{\delta}=\left\{r, z:\left[r-\left(r_{1}+r_{2}\right) / 2\right]^{2}+z^{2}=\left(r_{0}+\delta\right)^{2}, \varphi: 0 \leq \varphi \leq 2 \pi\right\}
$$

$T_{\delta}$ is the $\delta$-toroid, and axial conditions of symmetry

$$
\left.\frac{\partial \Phi(r, z)}{\partial r}\right|_{r=r_{1}+r_{0}}=0,\left.\quad \frac{\partial \Phi(r, z)}{\partial z}\right|_{z=0}=0 .
$$

It is obviously that conditions (38), (39) correspond to the neutron flex $\Phi=\left(\Phi_{r}, \Phi_{z}\right)$ for which

$$
\Phi_{r}=0, \quad \Phi_{z}=0
$$

The initial diffusion Equation (26) with boundary (16) and axial (17) conditions corresponds the flex $\Phi=\left(\Phi_{r}, \Phi_{z}, \Phi_{\varphi}\right)$, with components

$$
\Phi_{r}=0, \quad \Phi_{z}=0, \quad \Phi_{\varphi}=\Phi_{0}=\text { const. }
$$

The flex (40) corresponds to the absence of leakage in a stationary $(k=1)$ nuclear electric generator, to the presence of the zero parameter $(a=0)$ in Equation (26), and to satisfying of the neutron balance equation:

$$
F-A-R=0 \text {. }
$$

The provision of condition (41) means an important feature and advantage of toroidal vacuum-nuclear system-the almost complete circular motion of the streams within the toroidal active zone of the generator.

In condition (38) there is a parameter $\delta$ called the extrapolation length. We shall say a few words about his physical sense. It turns out that the neutron flex does not vanish on the boundary or the edge of the medium in which the reaction takes place. In a toroidal nuclear device of finite dimensions there is always, though insignificant, a leakage through this external boundary. This means that in the immediate vicinity of the boundary inside of active zone there should be neutrons that leave its limits. At the boundary, some of the neutrons leave outside and the other part diffuses inside and increases the value of the neutron flex. As a result of this, the neutron flex on the boundary doesn't equal zero but is supposed equal zero from the outside on some little distance $\delta$.

Let's consider this case in more detail. Suppose that the boundary condition (38) is satisfied, and on the toroid itself $T$ there is a small neutron leakage, i.e. $\Phi(r, z) \neq 0$, but

$$
\Phi(r, z)=\varepsilon_{\varphi}, \quad \forall r, z \in T,
$$

where $\varepsilon_{\varphi}>0$ is a small numerical parameter. 
Then, obviously, the small external transverse diffusion in Equation (26) corresponds that $a^{2}=a_{r}^{2}+a_{z}^{2}=\varepsilon^{2}$, where $\varepsilon>0$ is some small parameter. In fact, this means that

$$
\frac{R}{D}=\frac{k_{1}-k_{2}-k_{3}}{D} \sim \varepsilon^{2},
$$

where $k_{1}, k_{2}, k_{3}$ are the coefficients of flex proportionality in the "formation", "absorption", "reflection" of neutrons, respectively. Hence we have $a_{r}=\varepsilon_{r}, a_{z}=\varepsilon_{z} ; \varepsilon_{r}, \varepsilon_{z}>0$ are small parameters.

Further we can write for the solution (37):

$$
\cos \varepsilon_{z} z \sim 1, \quad C_{1} J_{0}\left(\varepsilon_{r} r\right)+C_{2} Y_{0}\left(\varepsilon_{r} r\right) \sim \frac{\varepsilon_{\varphi}}{Z_{0}} .
$$

Since for the Bessel and Neumann functions of zero order we have

$$
J_{0}(0) \sim 1, \quad Y_{0}(0) \sim-\infty,
$$

then it is naturally we can choose: $C_{1}=\varepsilon_{\varphi} / Z_{0}, C_{2}=0$.

Thus, the case of small transverse neutron diffusion with the indicated characteristics in the toroid is quite probable and can be considered as a kind of nuclear analogy of Arnold diffusion [12] across the stochastic layer.

In conclusion, we investigate the nonstationary case by limiting to a simple situation in which there are no delayed neutrons and $k>1$. The change in the neutron density with time is described by the relation $n(t)=n_{0} \exp \left(t / \tau_{0}\right)$, where $n_{0}$ is the neutron density in the initial moment of time, $\tau_{0}=T /(k-1)$ is the period. We assume from this that the neutron flex can be represented in the form

$$
\Phi(t)=\Phi_{0} \varphi(t), \quad \Phi(0)=\Phi_{0} \varphi(0)=\Phi_{0} \varphi_{0},
$$

where $\varphi(t)$ is a continuously differentiable function of time. Then the general diffusion equation can be written as follows

$$
\frac{\Delta \Phi+k_{e} \Phi}{T}=\frac{\mathrm{d} \Phi}{\mathrm{d} t},
$$

where the rate of change of the nonstationary neutron flex is proportional to the total "penetrating diffusion" $\Delta \Phi+k_{e} \Phi$ over the lifetime of one generation $T$. Here $k_{e}=k-1$ is the effective coefficient of reproduction.

It remains to substitute into Equation (42) $\Phi(t)=\Phi_{0} \varphi(t)$ and take into account that $\Delta \Phi=0$. We have

$$
\frac{\mathrm{d} \varphi(t)}{\mathrm{d} t}=\frac{k_{e} \varphi(t)}{T}
$$

from which

$$
\varphi(t)=\varphi_{0} \mathrm{e}^{t / \tau_{0}}, \quad \Phi(t)=\Phi_{0} \varphi_{0} \mathrm{e}^{t / \tau_{0}} .
$$

Note that due to the assignment of the flex $\Phi(t)$ takes place $\Delta \Phi=0$. However, it is not difficult to show that on the basis of the previous analysis, that in the case $\Phi(w, t)=\Phi(w) \varphi(t)$ where $w$ is the spatial variable, we have for the 
toroid $\Delta \Phi(w, t)=0$. Thus, we can conclude from this that the cases of neutron leakage from the active toroidal zone are excluded. The same reasoning can also be given for an ionite gas. Other coefficients and notations appear, but the essence, must be assumed, remains the same: the ionites in the toroid will practically not be subject to diffusion.

\section{Conclusions}

First of all, it should be noted once more that in this paper the problem of neutron multiplication in the nuclear fission process is solved in general form. It may be achieved owing to: 1) the introduction of the matrix of full neutron kinetics and the investigation of its algebraic properties; 2) the reducing of integrodifferential task to integral task for the effective solution of kinetic neutron equations.

Flex (40) corresponds to the absence of the leakage in stationary $(k=1)$ nuclear electric generator, the availability of zero parameter $(a=0)$ in Equation (26) and the carrying-out of neutron balance equation: $F-A-R=0$. The fulfillment of this condition means the important peculiarity and advantage of toroidal vacuum-nuclear systems, expressed into practically complete (without losses) circular motion of nuclear flexes inside of the toroidal active zone of nuclegen.

\section{References}

[1] Tertychny-Dauri, V.Yu. (2017) Solution of Nuclear Electrodynamics Equations Taking into Account $\beta$-Charged Eradiation. Open Access Library Journal, 4, e3794. https://doi.org/10.4236/oalib.1103794

[2] Tertychny-Dauri, V.Yu. (2017) Diffusion of Neutrons in the Toroidal Nuclear Electrogenerator. VI International Conference on Mathematical Modeling in Physical Sciences, Pafos, Cyprus, 28-31 August 2017, IOP Journal of Physics. Conference Series, 936, Article ID: 012007.

[3] Tertychny-Dauri, V.Yu. (2018) Nuclear Electromagnetic Generator: Mathematical Model on Toroidal Vacuum Scheme. Open Access Library Journal, 5, e4524. https://doi.org/10.4236/oalib.1104524

[4] Tertychny-Dauri, V.Yu. (2018) Nuclear Electromagnetic Generator: Introduction in Charge Algebra and Elements of Charge Kinetics. Open Access Library Journal, 5, e4614. https://doi.org/10.4236/oalib.1104614

[5] Harrer, J.M. (1963) Nuclear Reactor Control Engineering. D. Van Nostrand Company, Inc., Princeton, Toronto, London, New York.

[6] Keepin, G.R. (1965) Physics of Nuclear Kinetics. Addison-Wesley Publishing Company, London.

[7] Dementyev, B.A. (1973) Kinetics and Regulation of Nuclear Reactors. Atomizdat, Moscow. (In Russian)

[8] Bellman, R. (1960) Introduction to Matrix Analysis. McGraw-Hill Book Company, Inc., New York, Toronto, London.

[9] Gantmacher, F.R. (1967) The Theory of Matrices. Nauka, Moscow. (In Russian)

[10] Volterra, V. (1959) Theory of Functionals and of Integral and Integrodifferential 
Equations. Dover Publications, Inc., New York.

[11] Tertychny-Dauri, V.Yu. (2002) Adaptive Mechanics. Kluwer Academic Publishers, Dordrecht, Boston, London. https://doi.org/10.1007/978-94-007-0787-0

[12] Lichtenberg, A.J. and Lieberman, M.A. (1983) Regular and Stochastic Motion. Springer-Verlag, New York, Heidelberg, Berlin.

https://doi.org/10.1007/978-1-4757-4257-2 